\title{
Multiferroic based on nematic liquid crystal and nanoparticles
}

\author{
O.V. Kovalchuk ${ }^{1}$, T.M. Kovalchuk ${ }^{2}$, N.M. Kucheriavchenkova ${ }^{1}$, V.V. Sydorchuk ${ }^{3}$, S.V. Khalameida ${ }^{3}$ \\ ${ }^{1}$ Kyiv National University of Technologies and Design, Kyiv, Ukraine; \\ E-mail: akoval@knutd.com.ua \\ ${ }^{2}$ V. Lashkaryov Institute of Semiconductor Physics, NAS of Ukraine, Kyiv, Ukraine \\ ${ }^{3}$ Institute for Sorption and Problems of Endoecology, NAS of Ukraine, Kyiv, Ukraine
}

\begin{abstract}
Multiferroics stable in morphological parameters has been created by introducing ferromagnetic $\left(\mathrm{Fe}_{2} \mathrm{O}_{3}\right)$ and ferroelectric $\left(\mathrm{LiNbO}_{3}\right)$ nanoparticles with the concentration $0.3 \mathrm{wt} . \%$ into nematic liquid crystal $6 \mathrm{CHBT}$. Being based on the analysis of photos obtained in nonpolarized light of optical microscope, it has been shown that the average sizes of irregular geometric shape of aggregates of $\mathrm{Fe}_{2} \mathrm{O}_{3}$ nanoparticles range unities of micrometers and are one order higher than spherical aggregates of $\mathrm{LiNbO}_{3}$ nanoparticles. It has been shown that low-frequency dispersion of components for the complex permittivity is caused by near-electrode processes. The mechanism of this relaxation process has been ascertained, and its parameters for some of the samples have been determined. It has been shown that when introducing the nanoparticles the conductance of liquid crystal decreases, which may be caused by adsorption of ions (that carry charge through the liquid crystal) on the surface of the nanoparticles.
\end{abstract}

Keywords: multiferroic, nematic liquid crystal, nanoparticle, adsorption of ions, complex permittivity.

Manuscript received 10.03.16; revised version received 18.07.16; accepted for publication 13.09.16; published online 04.10.16.

\section{Introduction}

Multiferroics are the substances that can have both ferroelectric and ferromagnetic properties were practically obtained recently [1-3]. Theoretically the possibility of existence of such substances was ascertained much earlier. In purely scientific interest, in this class of materials it should be also expected that the presence of magnetic and electric ordering in these substances opens wide possibilities for their practical use in various fields of modern technology [3].

As it follows from the analysis of publications on multiferroics properties, solids were recently investigated. For the first time, the possibility to create multiferroics based on ferroelectric liquid crystal and Ni nanoparticles was demonstrated in [4]. The feature of this system was that the matrix (liquid crystal) itself had the ferroelectric phase.
The aim of this work was to study the possibility to create multiferroics by adding the nanoparticles of ferroelectric and ferromagnetic materials into nematic liquid crystal (NLC) that has neither ferromagnetic nor ferroelectric phase; to study changes in the dielectric properties of NLC under action of nanoparticles.

\section{Materials and methods of research}

To create multiferroic, we used nematic liquid crystal 6CHBT as a matrix. The thermotropic nematic liquid crystal 4-(trans-40-n-hexylcyclohexyl)-isothiocyanatobenzene (6CHBT) was synthesized and purified at the Institute of Chemistry, Military Technical University, Warsaw, Poland. The temperature of the phase transition from the isotropic liquid to the nematic phase was found to be $42.6{ }^{\circ} \mathrm{C}$. 
To provide magnetic properties, we introduced $\mathrm{Fe}_{2} \mathrm{O}_{3}$ nanoparticles. In this work, we used nanoparticles of $50 \mathrm{~nm}$ with the saturation magnetization of $85 \mathrm{~A} \cdot \mathrm{m}^{2} / \mathrm{kg}$. To reduce the effects of aggregation of the nanoparticles, they were coated with crosslinked dextrin. Since at the first stage of the research it was important to investigate stability and the possibility of interaction between these particles, the concentration of nanoparticles in NLC was chosen to be $0.3 \mathrm{wt} . \%$.

Ferroelectric properties were provided by introduction of the nanoparticles $\mathrm{LiNbO}_{3}$ into NLC. The nanoparticles were synthesized and studied at the Institute of Sorption and Problems of Endoecology, NAS of Ukraine by using the method described in [5]. Mixture of lithium carbonate and niobium pentoxide was subjected to dry mechanochemical activation at $850 \mathrm{rpm}$ using ball mill Pulverisette-7 (Fritsch, German). Activated mixture was calcined at $450{ }^{\circ} \mathrm{C}$ after milling. The size of $\mathrm{LiNbO}_{3}$ crystallites calculated using Sherrer's equation is $19.8 \mathrm{~nm}$. These crystallites are aggregates of the particles with a size of $90 \mathrm{~nm}$. The concentration of $\mathrm{LiNbO}_{3}$ nanoparticles in NLC, as in the case of $\mathrm{Fe}_{2} \mathrm{O}_{3}$ nanoparticles, was 0.3 wt. $\%$.

The nanoparticles were injected into NLC by mechanical stirring followed by sonication. Liquid crystal 6CHBT without/with nanoparticles was studied in the cells of sandwich type with transparent ITO electrodes. Previously onto the electrodes a polymer layer was coated and appropriately processed to create planar orientation of LC molecules. The thickness of the cells was $10 \mu \mathrm{m}$. Filling the cell with LC was performed using the capillary method at $5 \ldots 10^{\circ} \mathrm{C}$ higher temperature than that of phase transition 'nematic-isotrop'.

Morphology of the obtained structures was studied using an optical microscope in polarized and unpolarized light.

The dielectric properties of the sandwich cells were studied in the frequency range $10 \ldots 10^{6} \mathrm{~Hz}$ at $293 \mathrm{~K}$ by using the oscilloscopic method [6]. The amplitude measuring signal of the sinusoidal shape was $0.4 \mathrm{~V}$. Assuming that the equivalent circuit of the measuring cell is the resistance and capacitor connected in parallel, the values of resistance $R$ and capacitance $C$ for the samples at different frequencies were determined, and being based on these values and geometric dimensions of the cell we determined the imaginary $\left(\varepsilon^{\prime \prime}\right)$ and real $\left(\varepsilon^{\prime}\right)$ components of the complex dielectric conductivity, respectively. The conductivity of liquid crystal was measured from the magnitude of the resistance $R$ in the section of frequency dependence where the resistance does not depend on the frequency.

\section{Experimental results and analysis}

\subsection{Morphology of films}

The study of morphology inherent to the obtained film in polarized light of optical microscope has shown that the aggregates of nanoparticles can be most clearly seen in unpolarized light. Fig. 1 shows morphology of the samples obtained in unpolarized light for the following cases: $6 \mathrm{CHBT}+0.3$ wt. $\% \mathrm{Fe}_{2} \mathrm{O}_{3}(\mathrm{a}), 6 \mathrm{CHBT}+0.3$ wt. $\%$ $\mathrm{Fe}_{2} \mathrm{O}_{3}$ (b), 6CHBT +0.3 wt. $\% \mathrm{Fe}_{2} \mathrm{O}_{3}+0.3$ wt. $\%$ $\mathrm{LiNbO}_{3}$ (c).

Analyzing the data adduced in the figures, one can draw the following conclusions. Despite the fact that the $\mathrm{Fe}_{2} \mathrm{O}_{3}$ nanoparticles were coated with dextrin, the aggregates of these nanoparticles have an average size of one order of micrometers. Some of them, as seen from Fig. 1a, were larger than $1 \mu \mathrm{m}$. The aggregates of $\mathrm{LiNbO}_{3}$ nanoparticle both separately (Fig. 1b) and with $\mathrm{Fe}_{2} \mathrm{O}_{3}$ nanoparticles (Fig. 1c) have dimensions by the order less and close to the sizes of the nanoparticles themselves. The shape of aggregates is also significantly different. In the case of $\mathrm{Fe}_{2} \mathrm{O}_{3}$ nanoparticles, the aggregates have irregular geometric shape, and in the case of $\mathrm{LiNbO}_{3}$ nanoparticles it is close to spherical.

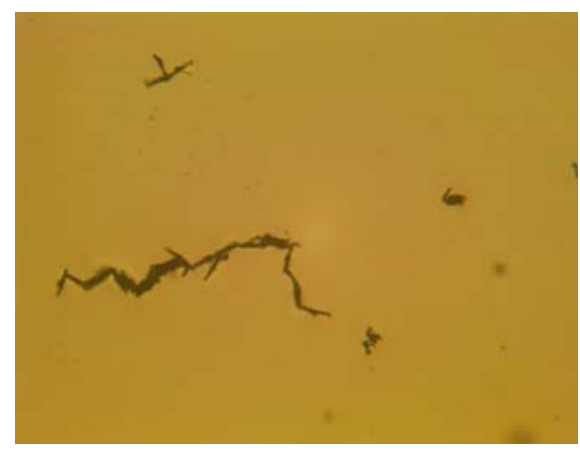

a)

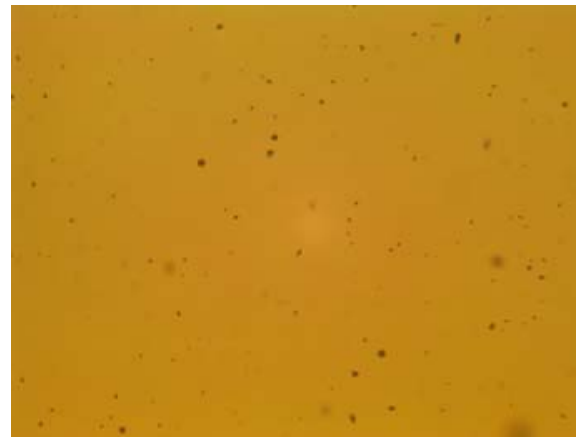

b)

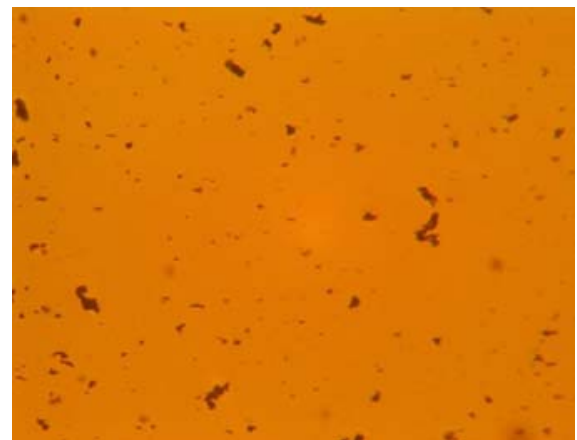

c)

Fig. 1. Morphology of the samples obtained in unpolarized light of optical microscope: 6CHBT +0.3 wt.\% $\mathrm{Fe}_{2} \mathrm{O}_{3}(\mathrm{a})$, $6 \mathrm{CHBT}+0.3$ wt. $\% \mathrm{Fe}_{2} \mathrm{O}_{3}$ (b), 6CHBT +0.3 wt. $\% \mathrm{Fe}_{2} \mathrm{O}_{3}+$ 0.3 wt. $\% \mathrm{LiNbO}_{3}$ (c). 
Another important conclusion follows from the analysis of Fig. 1c. It is seen that in the case when the ferromagnetic and ferroelectric nanoparticles were introduced into NLC, the aggregates of $\mathrm{Fe}_{2} \mathrm{O}_{3}$ nanoparticle are smaller in sizes (Fig. 1c) as compared to the samples where only $\mathrm{Fe}_{2} \mathrm{O}_{3}$ nanoparticles have been introduced (Fig. 1b).

From the analysis of Fig. 1c, one can also make a very important conclusion that $\mathrm{LiNbO}_{3}$ and $\mathrm{Fe}_{2} \mathrm{O}_{3}$ nanoparticles in NLC do not practically interact with each other (there is no aggregation of nanoparticles between them). This conclusion will be important to explain the dielectric spectra.

Our repeated (two months later) analysis of morphology of the samples revealed that significant changes in the distribution and shape of particles do not occur. That is, the obtained samples are stable over time.

\subsection{The impact of nanoparticles on the dielectric properties of NLC}

Fig. 2 shows the spectra of the real component of complex permittivity $\varepsilon^{\prime}$ for the samples: 6CHBT (1), $6 \mathrm{CHBT}+0.3$ wt. $\% \mathrm{Fe}_{2} \mathrm{O}_{3}(2), 6 \mathrm{CHBT}+0.3$ wt. $\%$ $\mathrm{LiNbO}_{3}(3)$ and $6 \mathrm{CHBT}+0.3$ wt. $\% \mathrm{Fe}_{2} \mathrm{O}_{3}+0.3$ wt. $\%$ $\mathrm{LiNbO}_{3}(4)$.

The analysis of these data shows that in the section of frequencies $f<10^{2} \mathrm{~Hz}$ dispersion of $\varepsilon^{\prime}$ is observed for all the samples. For the samples 6CHBT $+0.3 \mathrm{wt} . \%$ $\mathrm{LiNbO}_{3}$ and 6CHBT +0.3 wt. $\% \mathrm{Fe}_{2} \mathrm{O}_{3}+0.3$ wt. $\%$ $\mathrm{LiNbO}_{3}$, the analysis of this dispersion for the components of permittivity is difficulty carried out, because changes in $\varepsilon^{\prime}$ with decreasing frequency are very small. For the analysis of low-frequency dispersion, the dependences $\varepsilon^{\prime \prime}\left(\varepsilon^{\prime}\right)$ (the Cole-Cole diagram) were plotted and analyzed.

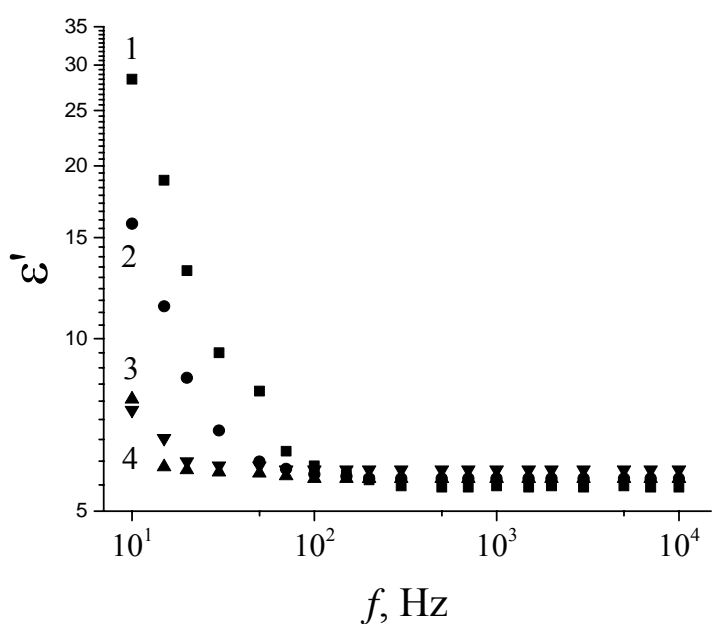

Fig. 2. Frequency dependences of the real component of complex permittivity $\varepsilon^{\prime}$ for the samples: 6CHBT (1), 6CHBT + 0.3 wt. $\% \mathrm{Fe}_{2} \mathrm{O}_{3}(2), 6 \mathrm{CHBT}+0.3$ wt. $\% \mathrm{LiNbO}_{3}$ (3) and $6 \mathrm{CHBT}+0.3$ wt. $\% \mathrm{Fe}_{2} \mathrm{O}_{3}+0.3$ wt. $\% \mathrm{LiNbO}_{3}$ (4). The sample thickness is $10 \mu \mathrm{m}$. The temperature is $293 \mathrm{~K}$.
The analysis of the Cole-Cole diagrams showed that they can be approximated by a semicircle with a low error. According to the theory of relaxation processes [7], this type of dispersion of permittivity value corresponds to the Debye dispersion and it is described by the relation:

$\varepsilon^{*}=\varepsilon_{\infty}+\frac{\varepsilon_{s}-\varepsilon_{\infty}}{1+i \omega \tau}$

where $\varepsilon^{*}$ is the complex permittivity, $\varepsilon_{\infty}$ - value of the dielectric constant at $f=\infty, \varepsilon_{s}-$ value of the dielectric constant at $f=0, \omega=2 \pi f-$ cyclic frequency, $\tau-$ relaxation time.

The values $\tau$ for the samples 6CHBT and 6CHBT + 0.3 wt. $\% \mathrm{Fe}_{2} \mathrm{O}_{3}$ are summarized in Table. The analysis of these results gives reason to believe that the value of $\tau$ for NLC is higher than hundred milliseconds and increases when adding $\mathrm{Fe}_{2} \mathrm{O}_{3}$ nanoparticles.

As it was shown in [8], the relaxation process that occurs at $f<10^{2} \mathrm{~Hz}$ can be explained on the basis of a two-layer Maxwell-Wagner polarization. In this case, one of the layers is a near-electrode section, in which for a given frequency band the charge transfer occurs due to fluctuations of dipoles of LC molecules within the angles corresponding to the fluctuations of the order parameter. The second layer is the bulk of the sample where charge transfer occurs due to ion transport. According to the theory of Maxwell-Wagner polarization the $\tau$ value should be inversely proportional to the conductance of LC. Therefore, it is precisely these changes in the electrical conductance of NLC when adding nanoparticles that can be a determining factor of dependence of the $\tau$ value on the content of nanoparticles.

Another parameter that can be estimated from the analysis of relaxation processes is the thickness of nearelectrode layer $l$ where charge transfer is mainly due to fluctuations of dipole molecules. To determine the $l$ value, we used the relation [9]:

$l=\frac{\varepsilon_{\infty} d}{2 \varepsilon_{s}}$

where $d$ is the sample thickness. The $l$ values for the samples 6CHBT ta $6 \mathrm{CHBT}+0.3$ wt. $\% \mathrm{Fe}_{2} \mathrm{O}_{3}$ are in Table. From the analysis of the obtained data, we can conclude that the addition of $\mathrm{Fe}_{2} \mathrm{O}_{3}$ nanoparticles to NLC leads to a small increase in the $l$ value.

Fig. 3 shows spectra of imaginary component of complex permittivity $\varepsilon$ " for the samples: 6CHBT (1), $6 \mathrm{CHBT}+0.3$ wt. $\% \mathrm{Fe}_{2} \mathrm{O}_{3}$ (2), $6 \mathrm{CHBT}+0.3$ wt. $\%$ $\mathrm{LiNbO}_{3}$ (3) and 6CHBT +0.3 wt. $\% \mathrm{Fe}_{2} \mathrm{O}_{3}+0.3$ wt.\% $\mathrm{LiNbO}_{3}(4)$. Common for all the samples is that at the frequencies $f>10^{2} \mathrm{~Hz}$ the $\varepsilon^{\prime \prime}$ value is inversely proportional to the frequency (data of the section in Fig. 3 are marked by line. This dependence corresponds to the case when the resistance of samples was independent of frequency. It is in these sections of frequency dependences of $\varepsilon$ " that we determined the conductance value from the ratio: 
$\sigma=\varepsilon_{0} \omega \varepsilon^{\prime \prime}$,

where $\varepsilon_{0}$ is the electric constant.

From the analysis of Fig. 3 and Table, one can make an important conclusion that the introduction of $\mathrm{Fe}_{2} \mathrm{O}_{3}$ and $\mathrm{LiNbO}_{3}$ nanoparticles (separately or together) into NLC leads to a decrease in conductance. The conductance of 6CHBT most decreases (by about 6 times) when introducing the $\mathrm{LiNbO}_{3}$ nanoparticles. The least decrease in conductance of NLC took place when introducing the $\mathrm{Fe}_{2} \mathrm{O}_{3}$ nanoparticles (by less than 2 times). Being based on the results of the analysis of sample morphology, it can be vastly explained greater aggregates of nanoparticles $\mathrm{Fe}_{2} \mathrm{O}_{3}$ than the $\mathrm{LiNbO}_{3}$ aggregates. Like to that in the work [10], as the main mechanism for reducing the conductance when introducing nanoparticles, we can consider adsorption of ions, which liquid crystal contains, and that are responsible for charge transfer on the $\mathrm{Fe}_{2} \mathrm{O}_{3}$ and $\mathrm{LiNbO}_{3}$ nanoparticles. Since the aggregates of $\mathrm{Fe}_{2} \mathrm{O}_{3}$ nanoparticles is much larger (here the surface area is small) than the $\mathrm{LiNbO}_{3}$ aggregates, then adsorption of ions on them occurs less effectively.

Table. Dependences of the parameters of near-electrode layers and the bulk of the homogeneous liquid crystal 6CHBT on the presence of $\mathrm{Fe}_{2} \mathrm{O}_{3}$ and $\mathrm{LiNbO}_{3}$ nanoparticles (separately or together). The sample thickness is $10 \mu \mathrm{m}$. The temperature is $293 \mathrm{~K}$.

\begin{tabular}{|l|c|c|c|}
\hline \multicolumn{1}{|c|}{ Sample } & $\tau, \mathrm{ms}$ & $l, \mathrm{~nm}$ & $\begin{array}{c}\sigma, \\
\mathrm{Ohm}^{-1} \mathrm{~m}^{-1}\end{array}$ \\
\hline 6CHBT & 52 & 165 & $5.0 \cdot 10^{-8}$ \\
\hline 6CHBT +0.3 wt.\% $\mathrm{Fe}_{2} \mathrm{O}_{3}$ & 67 & 200 & $3.0 \cdot 10^{-8}$ \\
\hline 6CHBT+0.3 wt.\% $\mathrm{LiNbO}_{3}$ & & & $8.4 \cdot 10^{-9}$ \\
\hline $\begin{array}{l}\text { 6CHBT }+0.3 \text { wt.\% } \% \mathrm{Fe}_{2} \mathrm{O}_{3}+ \\
\text { 0.3 wt.\% } \mathrm{LiNbO}_{3}\end{array}$ & & & $1.4 \cdot 10^{-8}$ \\
\hline
\end{tabular}

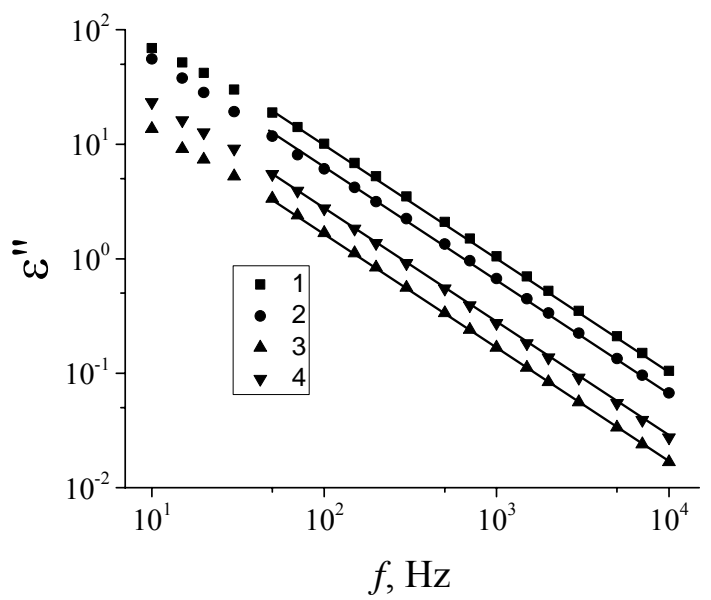

Fig. 3. Frequency dependences of the imaginary component of complex permittivity $\varepsilon$ " for the samples: 6CHBT (1), 6CHBT + 0.3 wt. $\% \mathrm{Fe}_{2} \mathrm{O}_{3}(2), \quad 6 \mathrm{CHBT}+0.3$ wt. $\% \mathrm{LiNbO}_{3}(3)$ and $6 \mathrm{CHBT}+0.3$ wt. $\% \mathrm{Fe}_{2} \mathrm{O}_{3}+0.3$ wt. $\% \mathrm{LiNbO}_{3}(4)$. The sample thickness is $10 \mu \mathrm{m}$. The temperature is $293 \mathrm{~K}$.
It was a significant decrease in conductance of the samples $6 \mathrm{CHBT}+0.3$ wt. $\% \mathrm{LiNbO}_{3}$ and $6 \mathrm{CHBT}+$ 0.3 wt. $\% \mathrm{Fe}_{2} \mathrm{O}_{3}+0.3$ wt. $\% \mathrm{LiNbO}_{3}$ that can be explained by the fact that for them it failed to estimate the parameters of near-electrode sections on the basis of the Cole-Cole diagrams. As we have shown in the works [9, $11]$, reducing the conductance of liquid crystal leads to a shift of section of dispersion $\varepsilon^{\prime}$ and $\varepsilon^{\prime \prime}$ toward lower frequencies.

Beside the process of aggregation of ions, it is evident that the reverse process is possible, namely, introduction of ions in addition to the existing ones in NLC with nanoparticles. For the concentrations of nanoparticles chosen for the research, this process is less effective than that of ion adsorption, but it is the fact that may explain why the conductance of the sample 6CHBT +0.3 wt. $\% \mathrm{Fe}_{2} \mathrm{O}_{3}+0.3$ wt. $\% \mathrm{LiNbO}_{3}$ is higher than that of $6 \mathrm{CHBT}+0.3$ wt. $\% \mathrm{LiNbO}_{3}$. For a reasonable answer to this question, it needs to perform the study with different concentrations of nanoparticles that will be the subject of our future research.

\section{Conclusions}

Multiferroic stable in time has been created by using the nematic liquid crystal $6 \mathrm{CHBT}$ and $\mathrm{Fe}_{2} \mathrm{O}_{3}$ and $\mathrm{LiNbO}_{3}$ nanoparticles. Being based on the analysis of morphology of the samples from photos obtained using unpolarized light of optical microscope, it has been shown that aggregates of $\mathrm{Fe}_{2} \mathrm{O}_{3}$ nanoparticles have average sizes of unities of micrometers and by an order exceeding the $\mathrm{LiNbO}_{3}$ aggregates. It has also shown that, unlike the almost spherical aggregates of $\mathrm{LiNbO}_{3}$ nanoparticles, the aggregates of $\mathrm{Fe}_{2} \mathrm{O}_{3}$ nanoparticles have irregular geometric shape.

It has shown that the low-frequency dispersion of components of complex permittivity of the samples observed for the frequencies $f<10^{2} \mathrm{~Hz}$ is caused by the Maxwell-Wagner polarization between the nearelectrode layers in which the conductance is provided vibrations of dipoles of molecules within the fluctuations of the order parameter and the bulk of the sample where the conductance is provided with transport of ions. For pure NLC and for $6 \mathrm{CHBT}+0.3 \mathrm{wt} . \% \mathrm{Fe}_{2} \mathrm{O}_{3}$ sample, the parameters of near-electrode process (the relaxation time and thickness of the layer where the charge transfer occurs due to fluctuations of dipoles of molecules) have been estimated and the influence of $\mathrm{Fe}_{2} \mathrm{O}_{3}$ nanoparticles on these parameters has been analyzed.

It has been shown that the conductance of the sample decreased with the introduction of nanoparticles. The biggest changes (6-fold decrease of conductance) were observed when introducing $0.3 \mathrm{wt} . \% \quad \mathrm{LiNbO}_{3}$ nanoparticles into 6CHBT LC. It has been concluded that the decrease in conductance of 6CHBT when introducing the nanoparticles is caused by adsorption of ions, which provide current in the liquid crystal, on the surface of nanoparticles. Since the sizes of aggregates of $\mathrm{LiNbO}_{3}$ nanoparticles were much smaller than those of 
$\mathrm{Fe}_{2} \mathrm{O}_{3}$ ones (and the effective surface area is much more) then their impact was much greater.

\section{Acknowledgements}

The authors are thankful to O.B. Bryk for the obtained $\mathrm{Fe}_{2} \mathrm{O}_{3}$ nanoparticles and S.V. Tomylko for investigation of the samples in the optical microscope.

\section{References}

1. W. Eerenstein, N.D. Mathur, J.F. Scott, Multiferroic and magnetoelectric materials // Nature, 442, p.759-765 (2006).

2. J.F. Scott, R. Blinc, Multiferroic magnetoelectric fluorides: Why are there so many magnetic ferroelectrics? // J. Phys.: Condens. Matter, 23, 113202 (2011).

3. A.P. Pyatakov, A.K. Zvezdin, Magnetoelectric and multiferroic media // Phys. Usp. 55, p. 557-581 (2012).

4. P. Ganguly, A. Kumar, K. Muralidhar, A.M. Biradar, Nanoparticles induced multiferroicity in liquid crystal // Appl. Phys. Lett. 108, 182905 (2016).

5. S. Khalameida, V. Sydorchuk, R. Leboda, J. Skubiszewska-Zięba, V. Zazhigalov, Preparation of nano-dispersed lithium niobate by mechanochemical route // J. Therm. Anal. Calorim. 115, p. 579-586 (2014)

6. A.J. Twarowski, A.C. Albrecht, Depletion layer in organic films: Low frequency measurements in polycrystalline tetracene // J. Chem. Phys. 70, p. 2255-2263 (1979).

7. F. Kremer, A. Schönhals, Broadband Dielectric Spectroscopy. Springer, 2003.

8. O. Yaroshchuk, A. Kovalchuk, R. Kravchuk, The interfacial dipole-to-dipole interaction as a factor of polar anchoring in the cells with planar liquid crystal alignment // Mol. Cryst. Liquid Cryst. 438, p. 195-204 (2005).

9. A.V. Koval'chuk, Low and infra-low dielectric spectroscopy liquid crystal-solid state interface. Sliding layers // Ukr. J. Phys. 41(10), p. 991-998 (1996).

10. S. Tomylko, O. Yaroshchuk, O. Kovalchuk, U. Maschke, R. Yamaguchi, Dielectric and Electrooptical properties of liquid crystals doped with diamond nanoparticles // Mol. Cryst. Liquid Cryst. 541, p. 35[273]-43[281] (2011).

11. A.V. Koval'chuk, Relaxation processes and charge transport across liquid crystal-electrode interface // J. Phys.: Condens. Matter, 13, p. 10333-10345 (2001). 